\section{Disease Activity Composite Indices in Patients with Rheumatoid Arthritis and Concomitant Fibromyalgia}

\section{To the Editor:}

We read with great interest the article by Coury, $e t a l^{1}$ comparing patients with rheumatoid arthritis (RA) and concomitant fibromyalgia (RAF). The ambiguous results of this study showed that patients with RAF had significantly higher disease activity measured by the 28 -joint Disease Activity Score (DAS-28) ${ }^{2}$, but significantly more severe joint destruction was revealed in patients with RA.

We examined 120 of our patients ( 29 men, 91 women) with RA according to the American College of Rheumatology (ACR) criteria $^{3}$ on the presence of concurrent fibromyalgia (FM). All the patients were examined by the same rheumatologist, assessing tender joint count (TJC), swollen joint count (SJC), and tender point count (TPC). Erythrocyte sedimentation rate (ESR), C-reactive protein (CRP), and the presence of erosions on hand and foot joint radiographs were also recorded. Disease activity was evaluated by DAS-28, which includes TJC, SJC, ESR, and patient general health by a $100 \mathrm{~mm}$ visual analog scale (VAS-GH), and also by newer composite indices: the Simplified Disease Activity Index (SDAI) ${ }^{4}$ and the Clinical Disease Activity Index (CDAI $)^{5}$. Functional disability was assessed using the Health Assessment Questionnaire.

FM diagnosis based on ACR criteria ${ }^{6}$ was established in $25(20.8 \%)$ patients (4 men, 21 women). Patients with RAF did not differ significantly in sociodemographic characteristics (age, RA duration, education, and marital status), inflammatory laboratory markers, joint erosion presence, and number of administered disease-modifying antirheumatic drugs (DMARD). All 3 composite indices were found to reach significantly higher values in RAF (Table 1). TJC and VAS-GH, which are subjectively influenced measurements, contribute mostly to the disease activity assessment differences in RA and RAF. However, the doctor's global health VAS, incorporated in SDAI and CDAI, was also eminently increased in patients with RAF in contrast to RA.

Monitoring disease activity in RA patients, followed by corresponding modification of DMARD therapy, is a cornerstone for successful treatment, with the aim to improve radiographic and functional outcomes in RA. Composite indices DAS-28, SDAI, and CDAI, widely used in daily clinical practice and clinical trials, are available instruments for such an assessment, but they can be insufficient to evaluate real inflammatory activity in cases of RA associated with chronic pain syndromes such as FM. The insufficiency of the mentioned indices results from measurements dependent on an individual patient's pain perception. Each rheumatologist should be aware of these limitations of currently used composite indices. Concomitant FM should be considered, especially in patients with RA with medium or high values of disease activity composite indices and marked divergence between subjective (pain evaluating) and objective (inflammatory) measurements (ESR, CRP, and SJC).

JAN TOMS, MD, Professor; TOMAS SOUKUP, MD, PhD; PETR BRADNA, MD, PhD; ZBYNEK HRNCIR, MD, PhD, Second Department of Medicine, Faculty of Medicine and University Hospital, Sokolska 581, Hradec Kralove 500 05, Czech Republic. Address correspondence to Dr. Toms, E-mail: toms.jan@seznam.cz

\section{REFERENCES}

1. Coury F, Rossat A, Tebib A, Letroublon MC, Gagnard A, Fantino $\mathrm{B}$, et al. Rheumatoid arthritis and fibromyalgia: A frequent unrelated association complicating disease management. J Rheumatol 2009;36:58-62.

2. van Gestel AM, Haagsma CJ, Van Riel PL. Validation of rheumatoid arthritis improvement criteria that include simplified joint counts. Arthritis Rheum 1998;41:1845-50.

3. Arnett FC, Edworthy SM, Bloch DA, McShane DJ, Fries JF, Cooper NS, et al. The American Rheumatology Association 1987 revised criteria for the classification of rheumatoid arthritis. Arthritis Rheum 1988;31:315-24.

4. Smolen JS, Breedveld FC, Schiff MH, Kalden JR, Emery P, Eberl $\mathrm{G}$, et al. A simplified disease activity index for rheumatoid arthritis for use in clinical practice. Rheumatology 2003;42:244-57.

5. Aletaha D, Nell VP, Stamm T, Uffmann M, Pflugbeil S, Machold $\mathrm{K}$, et al. Acute phase reactants add little to composite disease activity indices for rheumatoid arthritis: validation of a clinical activity score. Arthritis Res Ther 2005;7:R796-806.

6. Wolfe F, Smythe A, Yunus MB, Bennet RM, Bombardier C, Goldenberg D, et al. The American College of Rheumatology 1990 criteria for the classification of fibromyalgia. Report of the Multicenter Criteria Committee. Arthritis Rheum 1990;33:160-72.

J Rheumatol 2010;37:2; doi:10.3899/jrheum.090805

Table 1. Clinical and laboratory characteristics in patients with rheumatoid arthritis without fibromyalgia (RA) and with fibromyalgia (RAF).

\begin{tabular}{lccc}
\hline Characteristics & RA $(\mathrm{n}=95)$ & RAF $(\mathrm{n}=25)$ & $\mathrm{p}$ \\
\hline Sex ratio (male:female) & $25: 70$ & $4: 21$ & 0.431 \\
Age (yrs) & $55.9 \pm 13.6$ & $61.4 \pm 10.7$ & 0.063 \\
RA duration (yrs) & $10.9 \pm 9.1$ & $12.8 \pm 12.5$ & 0.771 \\
Tender point count & $3.5 \pm 3.4$ & $14.2 \pm 2.7$ & $<0.0001$ \\
Tender joint count & $2.87 \pm 3.6$ & $14.10 \pm 7.6$ & $<0.0001$ \\
Swollen joint count & $2.98 \pm 4.3$ & $4.92 \pm 3.9$ & 0.022 \\
ESR (mm/h) & $39.4 \pm 23.5$ & $28.5 \pm 29.2$ & 0.410 \\
C-reactive protein (mg/l) & $18.3 \pm 30.8$ & $17.5 \pm 24.7$ & 0.941 \\
Patient's global health VAS & $32.6 \pm 21.6$ & $60.4 \pm 15.9$ & $<0.0001$ \\
Doctor's global health VAS & $27.0 \pm 16.8$ & $45.5 \pm 10.7$ & $<0.0001$ \\
DAS-28 & $3.67 \pm 1.4$ & $5.35 \pm 1.1$ & $<0.0001$ \\
SDAI & $13.5 \pm 10.8$ & $31.8 \pm 10.9$ & $<0.0001$ \\
CDAI & $11.8 \pm 9.4$ & $29.6 \pm 10.7$ & $<0.0001$ \\
Health Assessment Questionnaire & $0.87 \pm 0.76$ & $1.83 \pm 0.64$ & $<0.0001$ \\
Joint erosion findings (\%) & 75.4 & 64.0 & 0.260 \\
\hline
\end{tabular}

ESR: erythrocyte sedimentation rate; DAS-28: 28-joint Disease Activity Score; SDAI: Simplified Disease Activity Index; CDAI: Clinical Disease Activity Index; VAS: visual analog scale. 